\title{
A CASE OF A FEMALE PRESENTING WITH RECURRENT EPISODES OF DIABETIC KETOACIDOSIS, DENOVO POST KIDNEY TRANSPLANT
}

\section{Hettiarachchi}

Department of Nephrology and Transplantation, Teaching Hospital Karapitiya, Galle

\section{ABSTRACT}

New onset diabetes after transplant (NODAT) occurs in a quarter of patients following renal transplants. NODAT is known to shorten the graft and patient survival. It is under diagnosed as a result of using fasting blood sugar or HbA1c as the investigation of choice as hyperglycemia tends to occur mainly post prandially.

Immunosuppressants are thought to play a major role in the causation of NODAT. Among those calcineurin inhibitors are thought to cause pancreatic beta cell exhaustion and corticosteroids to induce peripheral insulin resistance. By the time impaired glucose tolerance manifests, nearly $50 \%$ of pancreatic beta cells have become dysfunctional. Therefore if we chose to treat NODAT the traditional way using lifestyle modifications, oral hypoglycemics and then insulin, we may lose the opportunity to reverse the damage to pancreatic beta cells. Beta cell sparing using intensive Insulin therapy is the recommended treatment coupled with metformin in the prevention and treatment of NODAT.

Keywords: NODAT, calcineurin inhibitors, beta cell exhaustion, post prandial hyperglycemia

\section{CASE}

A 50 year old female with end stage renal disease due to chronic obstructive uropathy underwent a renal transplant in October 2016. Her immediate post transplant period was complicated by acute pancreatitis and bactereamia, requiring a prolonged hospital stay. She was commenced on triple immunosuppressants including prednisolone, tacrolimus and mycophenolate mofetil and continued. She was discharged on above medications with a satisfactory graft function. (serum creatinine $1.02 \mathrm{mg} / \mathrm{l}$ ) One and a half months post kidney transplant, she was admitted with polydipsia, polyuria and loss of appetite for 4 days duration. Sepsis was excluded and preliminary investigations revealed a random blood sugar of high index when tested with the glucometer, ketone bodies in urine and acidosis on arterial blood gas analysis. She was managed as diabetic ketoacidosis which has occurred denovo following kidney transplant. Throughout the period of admission, her graft function remained stable but she experienced severe muscle cramps and pain restricting movements of her lower limbs, following treatment with intravenous Insulin. Investigations revealed severe and prolonged hypokalemia which was managed with intravenous potassium chloride and later on with oral supplements. She was discharged on Insulin and later titrated and managed on oral hypoglycemic agents (OHA) with satisfactorily controlled blood sugar.

While she was on oral tolbutamide $500 \mathrm{mg}$ three times a day, she was readmitted at 6 months post kidney transplant with nonspecific symptoms and fever suggestive of sepsis. Her investigations revealed keto acidosis for the second time triggered by sepsis. Focus of sepsis could not be identified but urine; blood cultures and chest XR were negative. She received treatment with Insulin and empirical antibiotics and discharged when inflammatory markers were satisfactory and blood sugar was under control. Following this episode she was discharged on Insulin and her graft function continued to remain satisfactory.

\section{DISCUSSION}

NODAT occurs in nearly quarter of renal transplant recipients (RTR)(1). It is associated with shortened graft and patient survival and increased risk of infection and cardiovascular events. Even though post transplant hyperglycemia is common, there's 
still no consensus for the time frame to diagnose NODAT. Many cases of NODAT are missed due to the usage of fasting blood sugar as the diagnostic investigation as hyperglycemia tends to occur postpradially. HbA1c is also an erratic marker as hemoglobin level tends to be unstable during the early post transplant period. Oral glucose tolerance test captures abnormalities of hyperglycemia more accurately.

There are modifiable and non modifiable risk factors for NODAT such as age, ethnicity and family history. Among the modifiable risk factors immunosuppressant medications play a major role in the causation of NODAT. Calcineurine inhibitors (CNI) cause pancreatic beta cell exhaustion and reduced uptake of glucose by muscles and adipocytes. Tacrolimus when compared with cyclosporine is a better immunosuppressant as well as a drug with potent diabetogenic properties (2).mTOR inhibitors are also recognized as diabetogenic in studies involving RTR. Corticosteroids contribute to causation of NODAT by inducing peripheral insulin resistance. As a result there had been attempts at rapid withdrawal of steroids, the process of which was hindered by the increase in acute rejection episodes(3).

The traditional approach to management of NODAT has been to modify lifestyle factors, add OHA and then escalate to Insulin in a stepwise fashion. But nearly $50 \%$ of the pancreatic beta cells are dysfunctional by the time impaired glucose tolerance manifests (4).Thus by approaching above stepwise manner in the treatment, we may lose the opportunity to reverse the damage to pancreatic beta cells. It is well established that severe hyperglycemia in itself is toxic to pancreatic beta cells $(5,6)$.Therefore by normalizing plasma glucose early in the post transplant period, beta cells can be spared from the vicious cycle of glycotoxic injury and ultimately development of NODAT. Beta cell sparing with the usage of intensive insulin therapy was employed in post transplant hyperglycemia. It is proven in studies that treatment with insulin on patients who develop NODAT has made them become independent of insulin therapy while those who have been on OHA required ongoing anti diabetic treatment (7). Beta cell function can be preserved for at least 3.5 years with early and intensive therapy for 3 months with insulin and metformin. After 3 months of intensive therapy the subjects on continued insulin or OHA showed excellent glycemic control and retained beta cell function as measured by c peptide levels(8). Exogenous insulin has shown pathophysiological basis of resting the pancreatic beta cells in the prevention of NODAT. There is no evidence to suggest satisfactory and efficacious use of OHA alone in the treatment or prevention of NODAT (9). Lifestyle modifications should be started pre transplant in overweight patients in parallel with increased physical activity. Early and intense life style intervention in patients listed for transplant, rapid steroid withdrawal in low risk patients, dividing the daily dose of prednisolone, and changing from tacrolimus to cyclosporine when appropriate, could minimize the risk and impact of NODAT. Perhaps a very important step will be the early institution of insulin to rest the pancreatic beta cells. The transplant community and treating physicians have to brace itself to the changing paradigms in the prevention and management of NODAT.

\section{CONCLUSION:}

NODAT is a common occurrence among renal transplant recipients. As the development of this dampens the benefits of transplant it is of utmost importance for the treating physicians to be aware of the pathogenesis and the basis for treatment of this entity. The development of NODAT is mainly caused by the transplant medications in susceptible patients with risk factors. It should be understood that conventional stepwise approach to therapy in NODAT would be detrimental as beta cell exhaustion and apoptosis will make the patient dependent on anti diabetic treatment for the life time unless intensive therapy with exogenous insulin is used in the outset. It is proven in studies involving patients with renal transplants that early institution of Insulin rests the beta cells and prevents further damage by eliminating the glycotoxic effect. Such patients have been found to be independent of Insulin therapy after considerable periods of time and some can be completely taken off anti diabetic medication for the rest of their lives. Therefore exogenous Insulin should be the choice in treatment of post transplant hyperglycaemia in order to prevent development and continuity of NODAT. 


\section{REFERENCES:}

1. Kasiske BL, Snyder JJ, Gilbertson D. Diabetes mellitus after kidney transplantation in the United States.American Journal of Transplant 2003; 3(2):178-185

2. Vincenti F, Friman S, Scheuermann E. Results of an international, randomized trial comparing glucose metabolism disorders and outcome with cyclosporine versus tacrolimus. American Journal of Transplant2007; 7: 1506-1514

3. Pascual J, Zamora J, Galeano C. Cochrane Database Systematic Review 2009; (1):CD005632.

4. Abdul-Ghani MA, Tripathy D, DeFronzo RA. Contributions of beta-cell dysfunction and insulin resistance to the pathogenesis of impaired glucose tolerance and impaired fasting glucose. Diabetes Care 2006; 29:1130-1133

5. Zhang TZ, Li J, Yang L. Cytotoxic Role of Intermittent High Glucose on Apoptosis and Cell Viability in Pancreatic Beta Cells. Journal of Diabetes Research Volume 2014, Article ID 712781, 9 pages

6. Leahy JL, Bonner-Weir S, Weir GC. Beta-cell dysfunction induced by chronic hyperglycaemia. Current ideas on mechanism of impaired glucose-induced insulin secretion. Diabetes Care 1992; 15(3): 442-455

7. Hecking M, Haidinger M, Döller D. Early Basal Insulin Therapy Decreases NewOnset Diabetes after Renal Transplantation. Journal of American Society of Nephrology 2012; 23(4): 739749.

8. Harrison LB, Adams-Huet B, Raskin, P. $\beta$ Cell Function Preservation After 3.5 Years of Intensive Diabetes Therapy.Diabetes Care 2012; 35(7): 1406-1412

9. Sharif A, Hecking M, de Vries APJ. Proceedings From an International Consensus Meeting on Post transplantation Diabetes Mellitus: Recommendations and Future Directions. American Journal of Transplantation 2014; 14: 1992-2000 